\title{
Commentary: A Tablet-Based Assessment of Rhythmic Ability
}

\author{
Agnès Zagala ${ }^{1,2,3 *}$, Nicholas E. V. Foster ${ }^{1,2,3}$ and Simone Dalla Bella $a^{1,2,3,4 *}$ \\ ${ }^{1}$ International Laboratory for Brain, Music and Sound Research (BRAMS), University of Montreal, Montreal, QC, Canada, \\ ${ }^{2}$ Department of Psychology, University of Montreal, Montreal, QC, Canada, ${ }^{3}$ Centre for Research on Brain, Language and \\ Music (CRBLM), Montreal, QC, Canada, ${ }^{4}$ Department of Cognitive Psychology, University of Economics and Human \\ Sciences in Warsaw, Warsaw, Poland
}

Keywords: music, rhythm, movement, auditory-motor synchronization, assessment mobile technologies, individual differences

\section{A Commentary on:}

A Tablet-Based Assessment of Rhythmic Ability

OPEN ACCESS

Edited by:

Nicola Bruno,

University of Parma, Italy

Reviewed by:

Theodore Zanto,

University of California, San

Francisco, United States

${ }^{*}$ Correspondence:

Agnès Zagala

agnes.zagala@umontreal.ca

Simone Dalla Bella

simone.dalla.bella@umontreal.ca

Specialty section:

This article was submitted to Human-Media Interaction, a section of the journal Frontiers in Psychology

Received: 17 September 2020 Accepted: 14 June 2021 Published: 20 July 2021

Citation:

Zagala A, Foster NEV and Dalla Bella S (2021) Commentary: A Tablet-Based Assessment of Rhythmic Ability. Front. Psychol. 12:607676. doi: 10.3389/fpsyg.2021.607676
Zanto, T.P., Padgaonkar, N.T., Nourishad, A., and Gazzaley, A. (2019). A Tablet-Based Assessment of Rhythmic Ability. Front. Psychol. 10:2471. doi: 10.3389/fpsyg.2019.02471

\section{INTRODUCTION}

Humans are well-equipped to move along with auditory rhythms via finger or foot tapping, body swaying or walking (Leman et al., 2013; Sowiński and Dalla Bella, 2013). Individual differences in auditory-motor synchronization abilities (AMS) are observed in the general population (Repp, 2010; Sowiński and Dalla Bella, 2013; Palmer et al., 2014), and exacerbated by disorders (e.g., language/speech disorders, Lundetræ and Thomson, 2018; Ladanyi et al., 2020; Parkinson, Yahalom et al., 2004; Puyjarinet et al., 2019). Describing these individual differences can shed light on the cognitive mechanisms underlying the rhythm system in healthy and patient populations (Dalla Bella, 2020; Damm et al., 2020; Ladanyi et al., 2020).

Finger tapping to test AMS (Repp, 2005; Repp and Su, 2013) is used in test batteries like the Battery for the Assessment of Auditory Sensorimotor and Timing Abilities (BAASTA, Dalla Bella et al., 2017), and the Harvard Beat Assessment Test (H-BAT, Fujii and Schlaug, 2013). Tapping performance is typically measured in the lab with tapping pads or dedicated sensors, which afford high temporal precision $(\leq 1 \mathrm{~ms})$, but make the method quite unsuitable to be used outside the lab.

\section{USING MOBILE DEVICES FOR TESTING RHYTHMIC ABILITIES}

The portability of tablets and smartphones makes them an appealing solution for testing cognitive functions (Koo and Vizer, 2019), and rhythm abilities (tablet version of BAASTA; Puyjarinet et al., 2017; Bégel et al., 2018; Dauvergne et al., 2018). The study by Zanto et al. (2019) contributes to the demonstration that mobile technologies can serve purposefully for assessing AMS abilities. With their AMS task on tablet, Zanto et al. aimed at replicating outcomes of well-known AMS tasks, such as tapping to a metronome. The results are broadly consistent with previous studies, showing, 


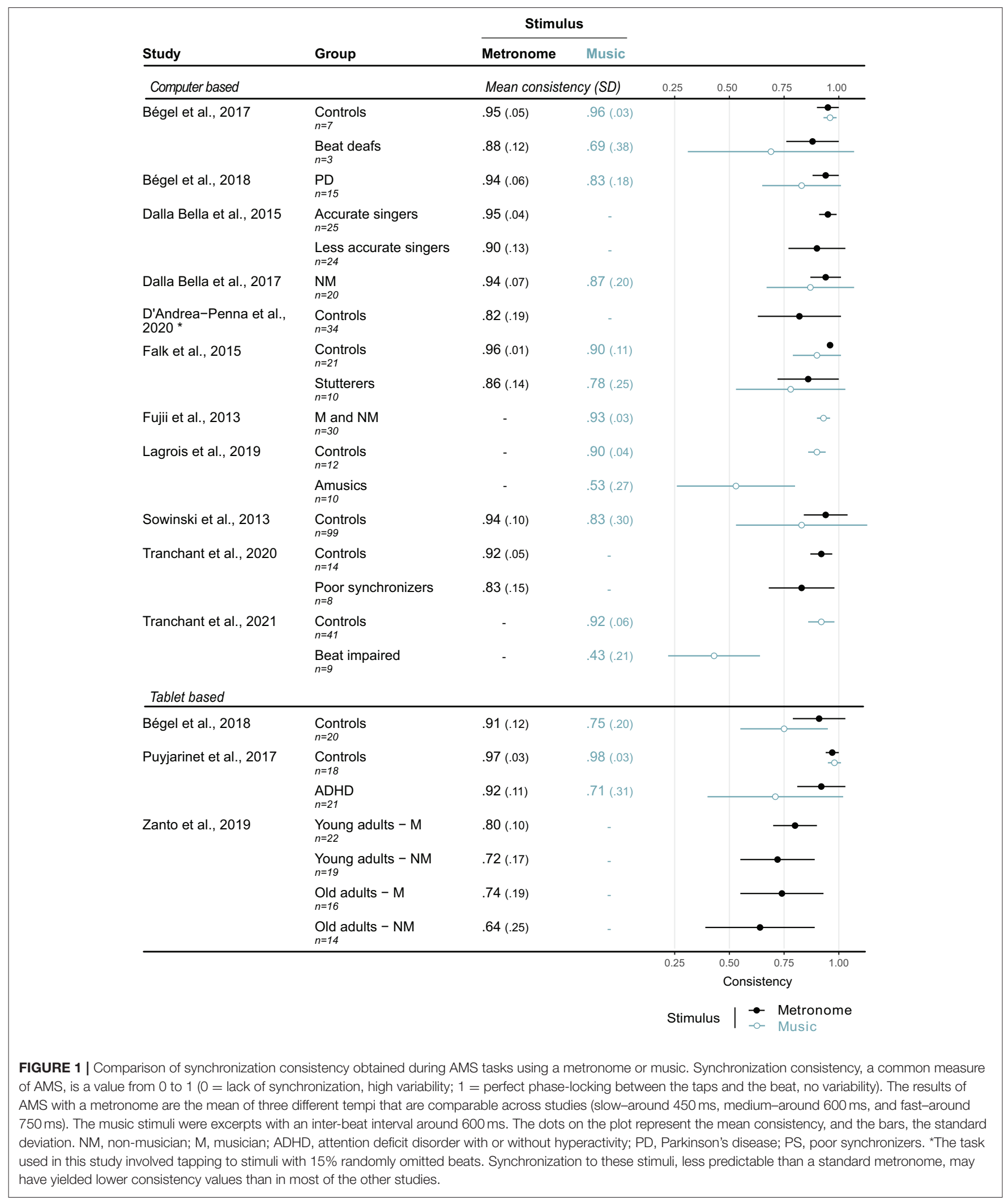

for example, that musicians are more consistent than nonmusicians in paced tapping (Franěk et al., 1991; Repp, 2010). Nevertheless, we notice that AMS performance (see Figure 1) is relatively low compared to other studies. This discrepancy may be linked to some of the limitations inherent in using touchscreen technology for tap detection. 


\section{LIMITATIONS AND INDICATIONS FOR FUTURE RESEARCH}

Timing inaccuracy in AMS tapping tasks on tablet can arise from: (1) a delay in the audio output, (2) the temporal uncertainty arising from the sampling rate of touch detection, and (3) the processing delay between the touch detection and the recording of a tap. Some of these limitations stem from the precision of the device touchscreen (sampling rate between 60 and $240 \mathrm{~Hz}$ ) which is much lower as compared to lab measurements $(1,000 \mathrm{~Hz}$ or more). Lower sampling rate imparts an unavoidable uncertainty about when the touch event occurred (Kousa, 2017). This variability (jitter) of error in individual touch events cannot be removed by subtracting an average delay. Because of this limitation, the participant's taps would appear more variable than their actual performance when measured in the lab. This hinders the capacity of the task to capture fine grained differences in AMS, and potentially to distinguish between good and poor synchronizers.

We compared the results from Zanto et al. (2019) with other in-lab studies and those using tablet devices (Figure 1), by taking synchronization consistency as a measure of variability in paced tapping (Fujii and Schlaug, 2013; Sowiński and Dalla Bella, 2013; Woodruff Carr et al., 2014). This measure corresponds to the vector length of the distribution of tap times within the inter-beat-interval, obtained with circular statistics. This measure shows high sensitivity to poor synchronization (e.g., Bégel et al., 2017; Lagrois and Peretz, 2019). Hence, synchronization consistency is a well-suited metric to assess the temporal precision of an AMS task, irrespective of constant latency in the task's technological implementation. It is apparent that non-musicians from Zanto et al.'s study generally obtained lower synchronization consistency (i.e., performed worse; mean consistency $=0.73$; range $=0.64-0.80)$ than healthy adults from all the other studies (mean consistency $=0.93$; range $=0.82-0.97$ ) considered here. Their results are sometimes comparable to or show poorer synchronization than individuals with rhythm disorders (Bégel et al., 2017, 2018; Puyjarinet et al., 2017; Lagrois and Peretz, 2019). The observed generally lower synchronization consistency is likely to reflect low timing precision of touchscreen devices, a fact that may hinder quantitative comparison with validated norms and other laboratory-based studies of synchronization consistency. However, the performance reported by Zanto et al. is more comparable to values in the literature when considering other

\section{REFERENCES}

Bégel, V., Benoit, C.-E., Correa, A., Cutanda, D., Kotz, S., and Dalla Bella, S. (2017). "Lost in time" but still moving to the beat. Neuropsychologia 94:22. doi: 10.1016/j.neuropsychologia.2016.11.022

Bégel, V., Verga, L., Benoit, C.-E., Kotz, S. A., and Dalla Bella, S. (2018). Testretest reliability of the Battery for the Assessment of Auditory Sensorimotor and Timing Abilities (BAASTA). Ann. Phys. Rehabil. Med. 61, 395-400. doi: 10.1016/j.rehab.2018.04.001 measures of tapping variability (e.g., standard deviation of asynchrony). In spite of these discrepancies, however, the precision afforded by Zanto et al.'s task is sufficient to distinguish musicians from non-musicians, and young from older adults, while providing high test-retest reliability. Thus, it may have general diagnostic value (e.g., for screening purposes). In addition, it is worth noting that Zanto's protocol also extends to visual and audio-visual synchronization, which is usually not tested by other batteries.

When synchronizing with audio stimuli, these issues with measurement precision on a tablet device can be solved by relying on an audio recording of the sound the taps produce when they reach the touchscreen. This solution, which is deviceindependent and capable of high temporal precision $(\leq 1 \mathrm{~ms})$ without requiring prior calibration, is already implemented in a tablet version of BAASTA (Dalla Bella and Andary, 2020). By recording the combined audio of stimulus and response, and resolving each during analysis, it bypasses possible sources of delay and jitter. This may explain why the tablet version of BAASTA successfully replicated the results previously obtained in the lab on a computer for AMS.

\section{CONCLUSION}

Mobile devices such as tablets or smartphones are very promising methods for screening AMS abilities. Solutions based on audio recording can compensate the current limitations of mobile touchscreens, thus reducing measurement uncertainty and matching the precision of laboratory measurement.

\section{AUTHOR CONTRIBUTIONS}

AZ, NF, and SDB: wrote the manuscript. All authors contributed to the article and approved the submitted version.

\section{FUNDING}

This research was supported by a Discovery Grant (RGPIN2019-05453) to SDB from the Natural Science and Engineering Research Council of Canada.

\section{ACKNOWLEDGMENTS}

We would like to thank Dr. Theodore Zanto, who shared data from his study with us. 
Dalla Bella, S., Berkowska, M., and Sowiński, J. (2015). Moving to the beat and singing are linked in humans. Front. Hum. Neurosci. 9:663. doi: 10.3389/fnhum.2015.00663

Dalla Bella, S., Farrugia, N., Benoit, C.-E., Begel, V., Verga, L., Harding, E., and Kotz, S. A. (2017). BAASTA: battery for the assessment of auditory sensorimotor and timing abilities. Behav. Res. Methods 49, 1128-1145. doi: 10.3758/s13428-016-0773-6

Damm, L., Varoqui, D., De Cock, V. C., Dalla Bella, S., and Bardy, B. (2020). Why do we move to the beat? A multi-scale approach, from physical principles to brain dynamics. Neurosci. Biobehav. Rev. 112, 553-584. doi: 10.1016/j.neubiorev.2019.12.024

Dauvergne, C., Bégel, V., Gény, C., Puyjarinet, F., Laffont, I., and Dalla Bella, S. (2018). Home-based training of rhythmic skills with a serious game in Parkinson's disease: usability and acceptability. Ann. Phys. Rehabil. Med. 61, 380-385. doi: 10.1016/j.rehab.2018.08.002

Falk, S., Müller, T., and Dalla Bella, S. (2015). Non-verbal sensorimotor timing deficits in children and adolescents who stutter. Front. Psychol. 6:847. doi: 10.3389/fpsyg.2015.00847

Franěk, M., Mates, J., Radil, T., Beck, K., and Pöppel, E. (1991). Finger tapping in musicians and nonmusicians. Int. J. Psychophysiol. 11, 277-279. doi: 10.1016/0167-8760(91)90022-P

Fujii, S., and Schlaug, G. (2013). The Harvard Beat Assessment Test (H-BAT): a battery for assessing beat perception and production and their dissociation. Front. Hum. Neurosci. 7:771. doi: 10.3389/fnhum.2013.00771

Koo, B. M., and Vizer, L. M. (2019). Mobile technology for cognitive assessment of older adults: a scoping review. Innov. Aging 3:igy038. doi: 10.1093/geroni/igy038

Kousa, M. (2017). Design, implementation and evaluation of a low-cost, high accuracy feedback latency measurement system (Master's thesis). Aalto University, Espoo, Finland. Retrieved from: http://urn.fi/URN:NBN:fi:aalto201712188209

Ladanyi, E., Persici, V., Fiveash, A., Tillmann, B., and Gordon, R. (2020). Is atypical rhythm a risk factor for developmental speech and language disorders? WIREs Cogn. Sci. 11:e1528. doi: 10.1002/wcs.1528

Lagrois, M.-É., and Peretz, I. (2019). The co-occurrence of pitch and rhythm disorders in congenital amusia. Cortex 113, 229-238. doi: 10.1016/j.cortex.2018.11.036

Leman, M., Moelants, D., Varewyck, M., Styns, F., van Noorden, L., and Martens, J.-P. (2013). Activating and relaxing music entrains the speed of beat synchronized walking. PLoS ONE 8:e67932. doi: 10.1371/journal.pone.0067932

Lundetræ, K., and Thomson, J. M. (2018). Rhythm production at school entry as a predictor of poor reading and spelling at the end of first grade. Read. Writ. 31, 215-237. doi: 10.1007/s11145-017-9782-9
Palmer, C., Lidji, P., and Peretz, I. (2014). Losing the beat: deficits in temporal coordination. Philos. Trans. R. Soc. Lond. B. Biol. Sci. 369:20130405. doi: 10.1098/rstb.2013.0405

Puyjarinet, F., Bégel, V., Gény, C., Driss, V., Cuartero, M.-C., Kotz, S. A., Pinto, S., and Dalla Bella, S. (2019). Heightened orofacial, manual, and gait variability in Parkinson's disease results from a general rhythmic impairment. NPJ Park. Dis. 5:19. doi: 10.1038/s41531-019-0092-6

Puyjarinet, F., Bégel, V., Lopez, R., Dellacherie, D., and Dalla Bella, S. (2017). Children and adults with attention-deficit/hyperactivity disorder cannot move to the beat. Sci. Rep. 7, 1-11. doi: 10.1038/s41598-017-11295-w

Repp, B. H. (2005). Sensorimotor synchronization: a review of the tapping literature. Psychon. Bull. Rev. 12, 969-992. doi: 10.3758/BF03206433

Repp, B. H. (2010). Sensorimotor synchronization and perception of timing: effects of music training and task experience. Hum. Mov. Sci. 29, 200-213. doi: 10.1016/j.humov.2009.08.002

Repp, B. H., and Su, Y.-H. (2013). Sensorimotor synchronization: A review of recent research (2006-2012). Psychon. Bull. Rev. 20, 403-452. doi: 10.3758/s13423-012-0371-2

Sowiński, J., and Dalla Bella, S. (2013). Poor synchronization to the beat may result from deficient auditory-motor mapping. Neuropsychologia 51, 1952-1963. doi: 10.1016/j.neuropsychologia.2013.06.027

Woodruff Carr, K., White-Schwoch, T., Tierney, A. T., Strait, D. L., and Kraus, N. (2014). Beat synchronization predicts neural speech encoding and reading readiness in preschoolers. Proc. Natl. Acad. Sci. U.S.A. 111, 14559-14564. doi: 10.1073/pnas.1406219111

Yahalom, G., Simon, E. S., Thorne, R., Peretz, C., and Giladi, N. (2004). Hand rhythmic tapping and timing in Parkinson's disease. Parkinsonism Relat. Disord. 10, 143-148. doi: 10.1016/j.parkreldis.2003.10.001

Zanto, T. P., Padgaonkar, N. T., Nourishad, A., and Gazzaley, A. (2019). A tablet-based assessment of rhythmic ability. Front. Psychol. 10:2471. doi: 10.3389/fpsyg.2019.02471

Conflict of Interest: The authors declare that the research was conducted in the absence of any commercial or financial relationships that could be construed as a potential conflict of interest.

Copyright (c) 2021 Zagala, Foster and Dalla Bella. This is an open-access article distributed under the terms of the Creative Commons Attribution License (CC BY). The use, distribution or reproduction in other forums is permitted, provided the original author(s) and the copyright owner(s) are credited and that the original publication in this journal is cited, in accordance with accepted academic practice. No use, distribution or reproduction is permitted which does not comply with these terms. 